\title{
SNF8, a member of the ESCRT-II complex, interacts with TRPC6 and enhances its channel activity
}

Robert Carrasquillo ${ }^{1,3}$,Dequan Tian ${ }^{2,3}$,Sneha Krishna ${ }^{1,3}$, Martin R Pollak ${ }^{1,3}$, Anna Greka ${ }^{2,3^{*}}$ and Johannes Schlöndorff , $^{1,3^{*}}$

\begin{abstract}
Background: Transient receptor potential canonical (TRPC) channels are non-selective cation channels involved in receptor-mediated calcium signaling in diverse cells and tissues. The canonical transient receptor potential 6 (TRPC6) has been implicated in several pathological processes, including focal segmental glomerulosclerosis (FSGS), cardiac hypertrophy, and pulmonary hypertension. The two large cytoplasmic segments of the cation channel play a critical role in the proper regulation of channel activity, and are involved in several protein-protein interactions.
\end{abstract}

Results: Here we report that SNF8, a component of the endosomal sorting complex for transport-II (ESCRT-II) complex, interacts with TRPC6. The interaction was initially observed in a yeast two-hybrid screen using the amino-terminal cytoplasmic domain of TRPC6 as bait, and confirmed by co-immunoprecipitation from eukaryotic cell extracts. The amino-terminal 107 amino acids are necessary and sufficient for the interaction. Overexpression of SNF8 enhances both wild-type and gain-of-function mutant TRPC6-mediated whole-cell currents in HEK293T cells. Furthermore, activation of NFAT-mediated transcription by gain-of-function mutants is enhanced by overexpression of SNF8, and partially inhibited by RNAi mediated knockdown of SNF8. Although the ESCRT-II complex functions in the endocytosis and lysosomal degradation of transmembrane proteins, SNF8 overexpression does not alter the amount of TRPC6 present on the cell surface.

Conclusion: SNF8 is novel binding partner of TRPC6, binding to the amino-terminal cytoplasmic domain of the channel. Modulating SNF8 expression levels alters the TRPC6 channel current and can modulate activation of NFAT-mediated transcription downstream of gain-of-function mutant TRPC6. Taken together, these results identify SNF8 as a novel regulator of TRPC6.

Keywords: Transient receptor potential, Calcium channel, Protein-protein interaction, Calcineurin-NFAT signaling

\section{Background}

Transient receptor potential canonical channel 6 (TRPC6) is one of seven members of the canonical transient receptor potential (TRPC) family of calcium-permeable cation channels that allow for an increase in intracellular calcium following activation of G-protein coupled receptors and receptor tyrosine kinases [1]. TRPC channel subunits assemble to form homo- and/or hetero-tetramers [2]. Each TRPC subunit contains intracellular amino and carboxyl-

\footnotetext{
*Correspondence: greka.anna@mgh.harvard.edu; jschlond@bidmc.harvard.edu ${ }^{2}$ Department of Medicine, Nephrology Division, Massachusetts General Hospital, 149 13th Street, Room 8.102, Boston, MA 02114, USA

'Division of Nephrology, Beth Israel Deaconess Medical Center, Research North 304B, 99 Brookline Ave, Boston, MA 02215, USA

Full list of author information is available at the end of the article
}

terminal domains flanking six transmembrane domains; the intracellular sequences host canonical protein-protein interaction domains, including ankyrin-like repeats and a coiled-coil region in the amino-terminal cytoplasmic domain, and carboxyl-terminal TRP box, CIRB (calmodulin/ IP3 receptor binding) sequence, and a coiled-coil region [3]. Multiple proteins have been shown to bind to TRPC6, including $\mathrm{BK}_{\mathrm{Ca}}$ [4], calmodulin [5-7], FKBP12 [8], Fyn [9], IP3 receptor [7,10], MxA [11], the $\mathrm{Na}+\mathrm{K}+$-ATPase pump [12], PLCY [13], podocin [14,15], PTEN [16], and RNF24 [17]. In addition, TRPC6 channel activity can be modulated by tyrosine $[9,13]$ and serine/threonine phosphorylation [18-21], and by binding of phosphoinositides $[6,22,23]$. However, how these various post-translational

\section{Biomed Central}


modifications and interacting partners ultimately alter channel activity has not been fully elucidated.

TRPC6 has been implicated in several physiological and pathophysiological functions in the kidney, heart and vasculature. Mutations in TRPC6 are a cause of familial, autosomal-dominant, adult-onset focal segmental glomerulosclerosis (FSGS), a form of proteinuric renal disease [15,24], and increased TRPC6 expression has been observed in acquired proteinuric kidney disease $[25,26]$. In contrast, TRPC6-deficient mice show mild protection against angiotensin II mediated glomerular damage [27]. The majority of FSGS-associated mutations appear to be gain-of-function, with increased channel current amplitudes and reduced current decay having been reported [15,24,28,29]. As all currently identified FSGS disease-associated TRPC6 mutations map to the intracellular domains of the channel, it has been hypothesized that these mutations may act by disrupting the binding of regulatory proteins to TRPC6, with the slit diaphragm protein nephrin proposed as one such protein [13]. How enhanced TRPC6 activity leads to renal disease, though, remains unknown. In contrast, in the heart upregulation of TRPC6 has been linked to the development of pathologic cardiac hypertrophy through activation of the calcineurin-NFAT pathway [30,31]. Finally, examinations of TRPC6-deficient mice have suggested roles in hypoxic pulmonary vasoconstriction [32], the development of pulmonary edema in response to ischemia-reperfusion injury [33], and platelet function [34].

To discover novel binding partners of TRPC6, we performed a yeast two-hybrid screen using the aminoterminal domain of TRPC6 as bait and isolated SNF8 as a TRPC6 binding partner. SNF8 is the mammalian counterpart to yeast VPS22, a component of the ESCRT-II endosomal trafficking complex required for the endocytosis and lysosomal degradation of transmembrane proteins [35-37]. The SNF8-TRPC6 interaction was mapped to the first 107 residues of the $\mathrm{N}$-terminus of TRPC6, and was confirmed by co-immunoprecipitation as well as immunofluorescence microscopy. Additionally, overexpression and RNAi-mediated knockdown of SNF8 in tissue culture reveals a modulatory effect on wild-type and mutant TRPC6 channel activity as evidenced by whole-cell electrophysiology studies and transcriptional luciferase reporter assays.

\section{Results}

SNF8 binds to the amino-terminus of TRPC6 in the Yeast Two-Hybrid System

The amino-terminal cytoplasmic domain of human TRPC6 (amino acids 1-406) was used as bait in a yeast two-hybrid screen to identify potential TRPC6 interacting proteins in a human kidney cDNA library. On the basis of both nutritional selection and $\beta$-galactosidase activity, 10 clones of SNF8 (also known as EAP30 and VPS22) were isolated; all clones included the entire coding sequence of SNF8 and varying lengths of 5/ UTR. Full-length SNF8 fused to the Gal4 activation domain was able to interact with the aminoterminal cytoplasmic domain, but not the carboxylterminal cytoplasmic domain of TRPC6 in the yeast two-hybrid system as assessed by growth on histidine and adenine free media (Figure 1) and $\beta$-galactosidase activity (data not shown). Using truncation mutants of the amino-terminal of TRPC6, it was found that amino acids 1-107, containing the sequence prior to the ankyrin repeats, are necessary and sufficient for this interaction (Figure 1).

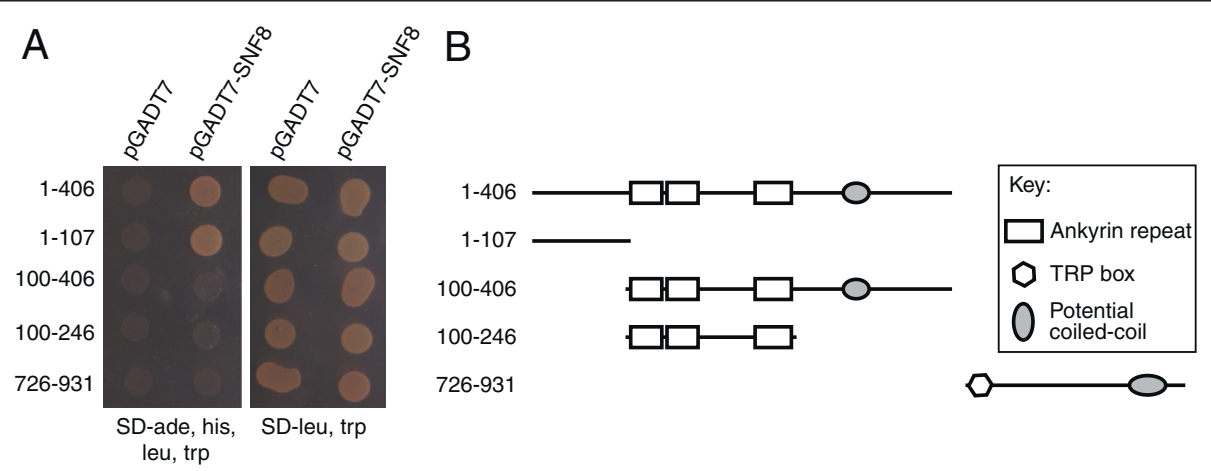

Figure 1 SNF8 binding to TRPC6 in the two-hybrid assay. A. Yeast carrying the pGBKT7 plasmid encoding for the Gal4 DNA binding domain fused to the indicated TRPC6 amino acids, and either pGADT7 (encoding for the GAL4 activation domain alone) or pGADT7-SNF8 (expressing full-length SNF8 fused to the GAL4 activation domain) were grown on high stringency selective media, SD-ade, his, leu, trp (left panel), to assess for protein-protein interaction, or on SD-leu, trp (right panel) as a control. B. Schematic of TRPC6 structure indicating the location of various structural domains relative to the TRPC6 constructs used in the yeast-two hybrid assay. 


\section{TRPC6 and SNF8 interact in mammalian cells}

To confirm that the interaction between TRPC6 and SNF8 can occur in a cellular context, cells stably expressing FLAG-tagged TRPC6 under a tetracycline inducible promoter were transfected with HA-tagged SNF8. TRPC6 was immunoprecipitated with the FLAG M2 monoclonal antibody, and the presence of SNF8 in the isolated immune complexes was assessed by Western blot using an anti-HA antibody (Figure 2A). HA-SNF8 was detected in the immunoprecipitated material only when co-expressed with FLAG-TRPC6. We have not been able to confirm the interaction by coimmunoprecipitation of endogenous SNF8 and TRPC6,

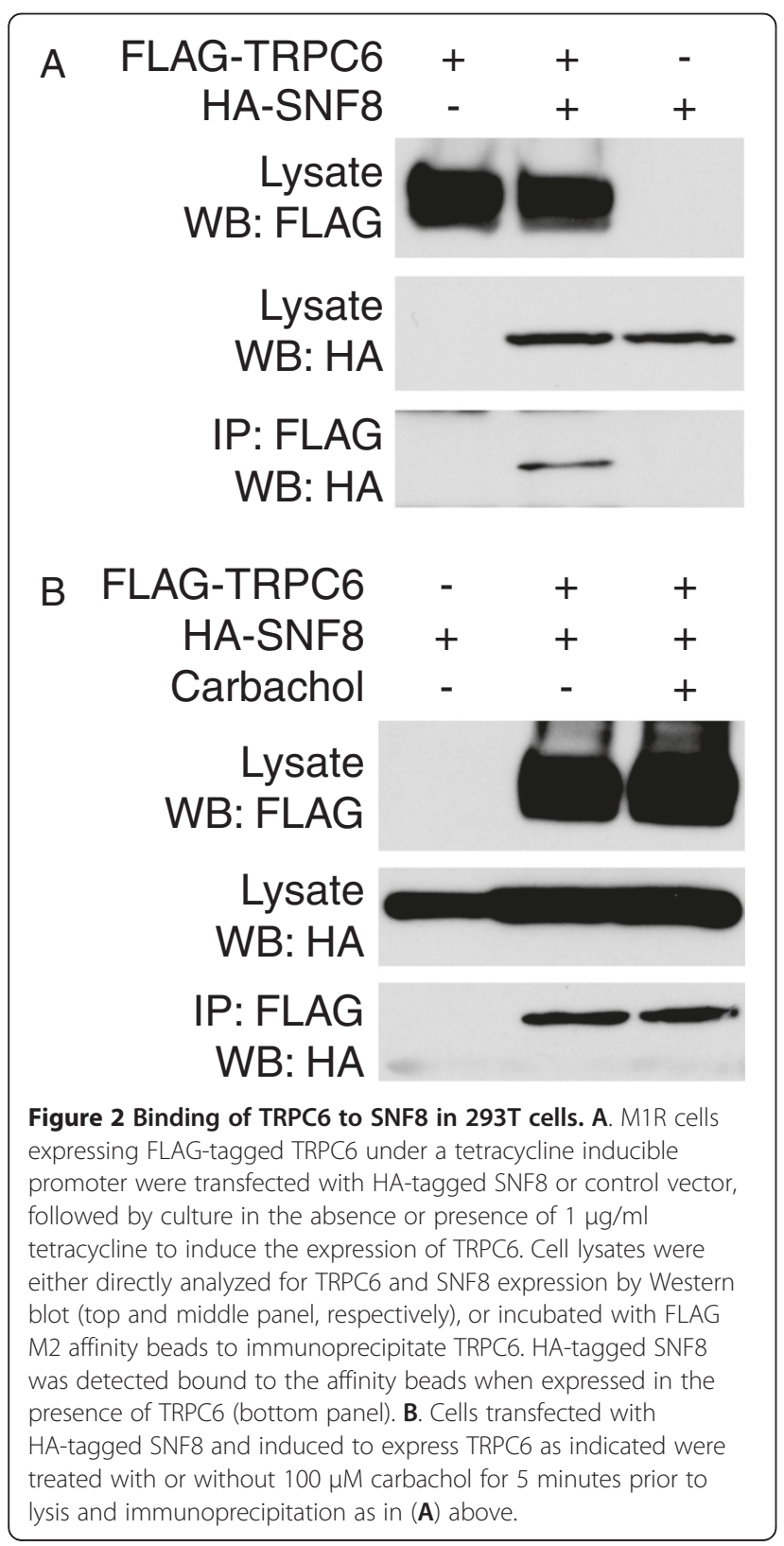

but this may be due at least in part due to the limited availability of high quality antibodies to detect endogenous SNF8 (data now shown) or a relatively low affinity interaction between the two proteins.

To assess if the interaction between the two proteins might be influenced by TRPC6 channel activation, cells co-expressing both proteins were treated with or without carbachol to activate TRPC6 prior to cell lysis and immunoprecipitation. No notable difference in the amount of SNF8 in the immune complexes was seen upon carbachol stimulation (Figure 2B). This suggests that channel activity or calcium influx is not required for the establishment of this interaction.

To further confirm the potential interaction between SNF8 and TRPC6, the localization of the two overexpressed proteins was determined by immunofluorescence microscopy. Overall, there was little colocalization of the two signals, though small punctate regions suggesting areas of colocalization of the two proteins were seen in most cells examined (Figure 3 ).

\section{SNF8 enhances TRPC6-mediated current}

To address whether SNF8 may play a role in the regulation of TRPC6 activity, we performed electrophysiology experiments in the whole cell configuration in HEK293T cells expressing the muscarinic M1 acetylcholine receptor and either TRPC6 and SNF8, or TRPC6 alone. Activation of the M1 receptor with carbachol resulted in a whole cell current with a characteristic current-voltage relationship for TRPC6: a small inward and a steeply outwardly rectifying component. Co-expression of SNF8 led to increased whole cell current amplitude in both the inward and outward directions (Figure $4 \mathrm{~A}$ and $\mathrm{C}$ ). The ability of SNF8 to enhance TRPC6-mediated currents extended to channels containing an FSGS-associated mutation, E897K (Figure 4B and C). This mutation was previously characterized as a gain-of-function mutation leading to increased whole cell current amplitude compared to wild-type controls [15].

We attempted to ascertain whether the effect of SNF8 on TRPC6 was dependent on direct binding of the two proteins. A TRPC6 construct lacking the amino terminal 106 amino acids (FLAG-TRPC6 107-931), required for SNF8 binding in the two hybrid assay, expressed at significantly lower levels than full-length TRPC6, though it was capable of forming multimers, based on its ability to co-immunoprecipitate full-length HA-tagged TRPC6 (Figure 5A). However, in contrast to full length TRPC6, the truncated protein failed to generate current in response to OAG stimulation (Figure 5B-D), preventing us from addressing whether the effect of SNF8 on TRPC6 currents was dependent on binding at this site. Similarly, TRPC6 107-931 failed to generate significant current after stimulation with carbachol (data not shown). 

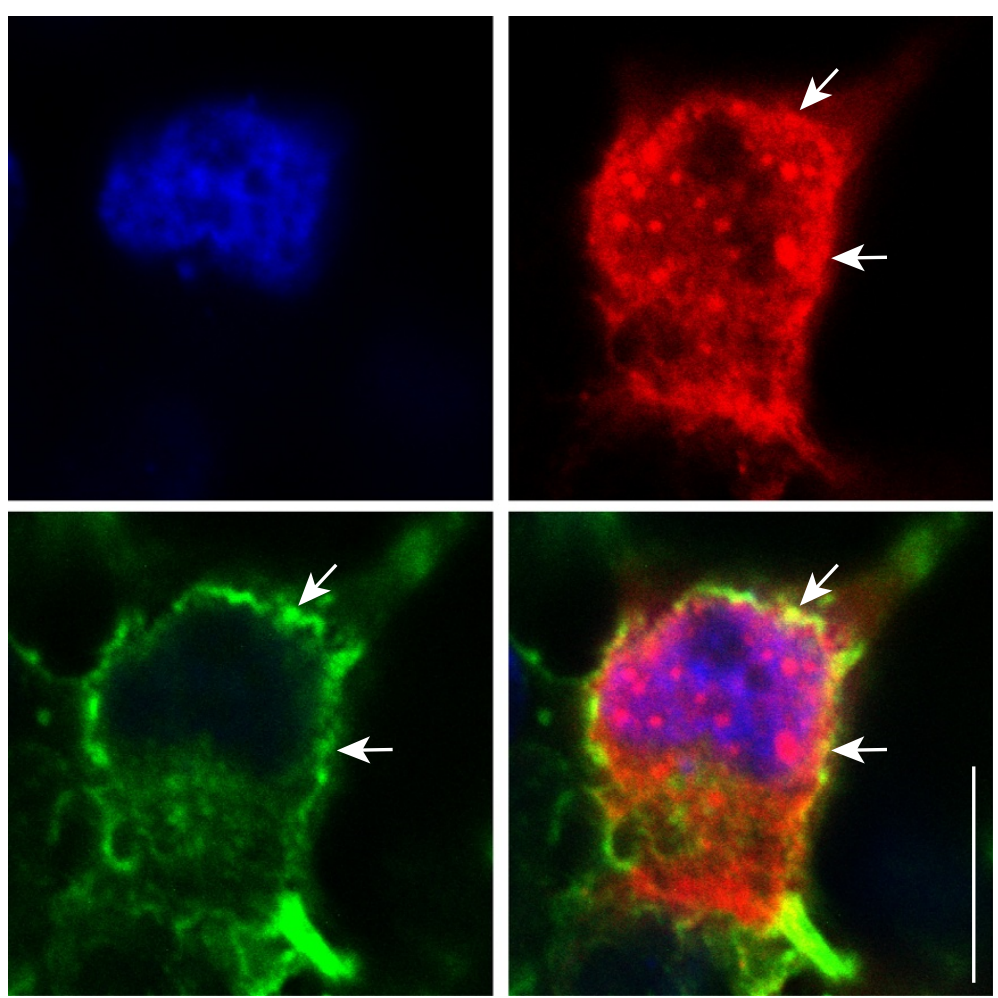

Figure 3 Immunofluorescence localization of TRPC6 and SNF8. M1R cells expressing FLAG-TRPC6 and HA-SNF8 were fixed and stained as follows: TRPC 6 was detected with the anti-FLAG M2 mouse monoclonal antibody and an Alexa488 conjugated anti-mouse secondary (bottom left); SNF8 was detected with an anti-HA rabbit monoclonal antibody and a Cy3 conjugated anti-rabbit secondary (top right). Nuclei were counterstained with DAPI (top left). A merged image (bottom right) demonstrates partial localization of TRPC6 and SNF8 in punctuate structures (arrows). $\mathrm{Bar}=10 \mu \mathrm{m}$.

\section{Modulating SNF8 levels alters NFAT-mediated transcription downstream of mutant TRPC6}

We have previously demonstrated that several FSGSassociated mutations in TRPC6 lead to activation of calcineurin-NFAT signaling and NFAT-mediated transcription in the absence of exogenous stimuli [38]. In light of the ability of SNF8 to enhance TRPC6-mediated whole cell currents, we examined the ability of SNF8 overexpression to affect TRPC6 mediated activation of NFAT-mediated transcription (Figure 6A). Overexpression of SNF8 in cells expressing either the R895C or E897K mutant TRPC6 channels led to an increase in NFAT-mediated transcription under unstimulated conditions. In contrast, in cells expressing wild-type TRPC6, which does not activate NFAT-mediated transcription under unstimulated conditions, SNF8 overexpression did not lead to activation of NFAT-driven transcription. Unfortunately, known activators of TRPC6, including carbachol and OAG, are able to substantially increase NFAT-mediated transcription in $293 \mathrm{~T}$ cells even in the absence of exogenously expressed TRPC6 [38]. Therefore, we have not been able to ascertain whether overexpression of SNF8 is able to enhance NFAT activation by wild-type TRPC6 after the channel has been activated.
Taken together with the electrophysiologic data, these results suggest that SNF8 can enhance TRPC6-mediated currents and downstream signaling pathways, but is not sufficient to activate the channel.

Most of our current knowledge regarding SNF8 relates to its function as part of the ESCRT-II complex involved in transmembrane protein trafficking into multivesiculated bodies $[37,39,40]$. We therefore hypothesized that overexpression of SNF8, rather than increasing ESCRT-II activity, might act in a dominant negative fashion by providing monomeric SNF8 to compete with intact ESCRT-II complex for TRPC6 binding. To address this possibility, we made use of a short hairpin RNA expressing plasmid to knock down SNF8 protein levels in M1R cells. Introducing the SNF8 shRNA expressing plasmid into cells expressing R895C mutant TRPC6 led to a modest decrease in activity from an NFAT-responsive luciferase reporter construct, compared to cells transfected with a control shRNA plasmid (Figure 6C). Expression of the SNF8 shRNA did not significantly alter basal NFATmediated transcription in control cells (WT uninduced and R895C uninduced) or expressing wild-type TRPC6 (WT induced). Western blot analysis confirmed a decrease in endogenous SNF8 levels with the SNF8 shRNA 


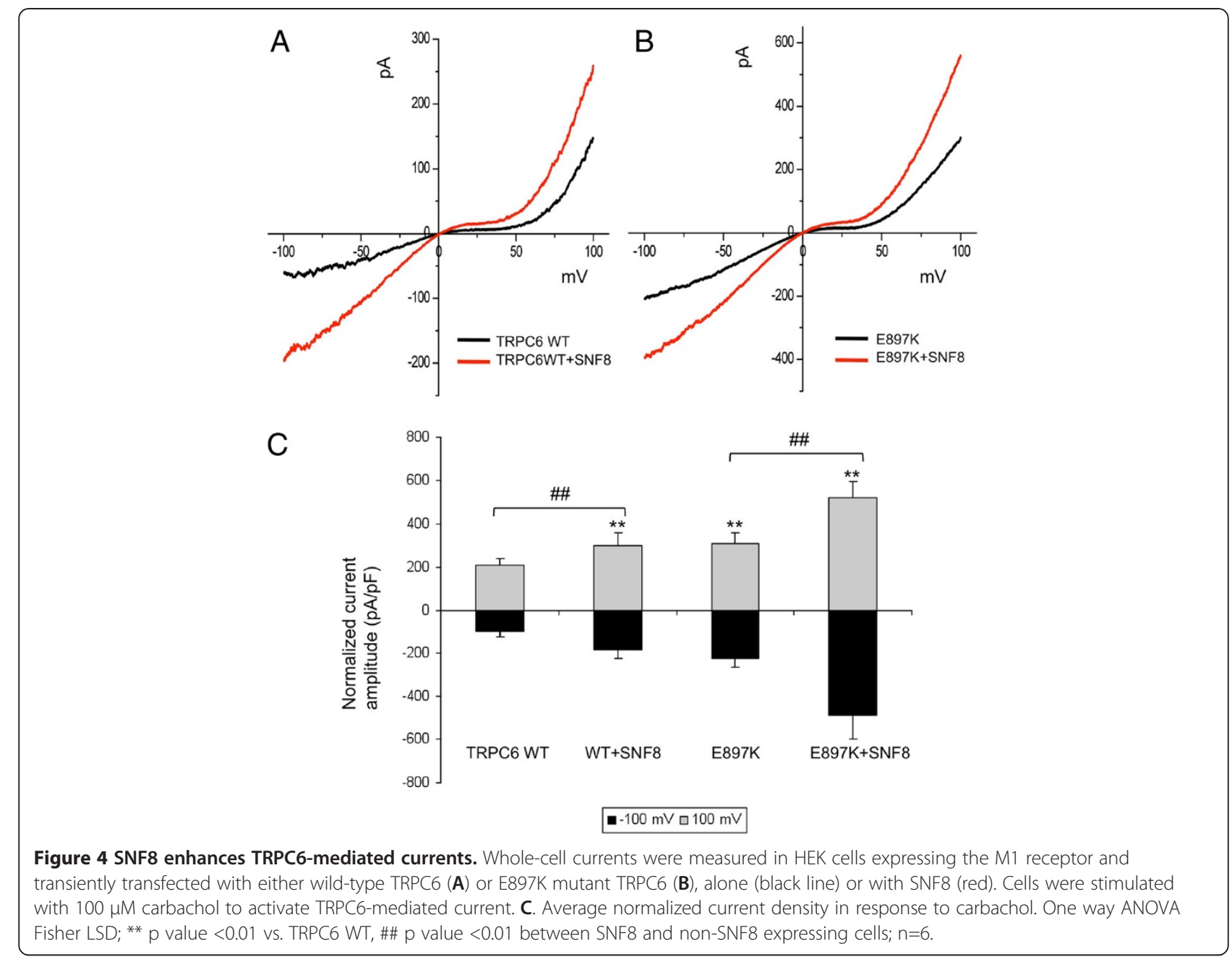

expression plasmid compared to control (Figure 6D). These results suggest that: 1 . the effects of overexpressed SNF8 on TRPC6 channel activity and signaling are not mediated by a dominant negative effect, and 2. while SNF8 may enhance TRPC6 activity, it is likely not required for channel activity given the only modest decrease in NFAT-reporter activity in response to SNF8 knockdown in R895 expressing cells. Confirmation of this later point will require the development of cells completely devoid of SNF8.

SNF8 overexpression does not alter TRPC6 content on the cell surface or in detergent resistant membranes

Several TRP channels, including TRPC6, have been reported to be regulated by altering their cell surface expression through regulated endo- and exocytosis $[13,41,42]$. Mutations in snf8 have been reported to affect the transport kinetics of Ena1p, a sodium pump in yeast [43]. We therefore wished to examine whether SNF8 overexpression might alter TRPC6 function by modulating the amount of channel expressed on the plasma membrane. M1R cells were transiently transfected with expression plasmids for FLAG-TRPC6 and HA-SNF8, either in combination, or alone with control plasmid. After surface biotinylation of the cells, the amount of TRPC6 bound to streptavidin beads (representing cell surface associated protein) was compared to total TRPC6 in whole cell lysates (Figure 7). Coexpression of SNF8 did not appreciably alter the amount of TRPC6 bound to the streptavidin beads compared to total TRPC6 levels. Interestingly, small amounts of SNF8 could also be detected in material bound to streptavidin beads (lower panels), consistent with its recruitment to transmembrane proteins, including plasma membrane proteins. However, the amount of SNF8 pulled down by streptavidin beads was not appreciably altered by the presence or absence of TRPC6, suggesting that cell surface proteins other than TRPC6 were largely responsible for this effect.

Several TRPC proteins have been reported to partition into detergent-resistant membranes [44-50], and TPRC1 binds to caveolin-1, a lipid raft-associated protein 


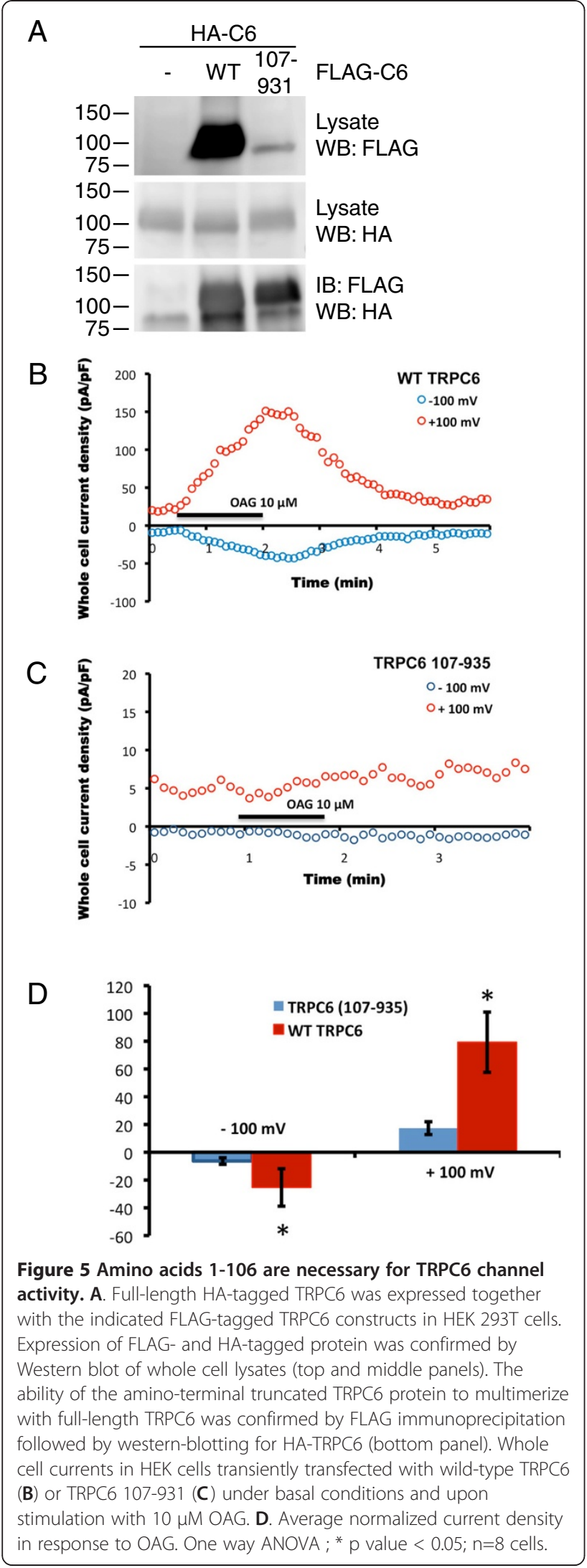

[45,47-49,51-53]. Furthermore, STIM1 overexpression was reported to shift TRPC1 into lipid rafts, thereby altering its channel properties [52,54,55]. We therefore examined whether SNF8 may alter the amount of TRPC6 present in detergent-resistant membranes. In control cells, only a very small fraction of TRPC6 (Figure 8A, top panel) was detected in the fractions containing detergent-resistant membranes, as assayed by the presence of the lipid raft marker caveolin-1 (Figure 8A, bottom panel), similar to previously reported results $[46,50]$. The amount of total TRPC6 found in the low density fractions did not change appreciably when HASNF8 was overexpressed (Figure 8B). A small amount of SNF8 was also detected in the detergent resistant membranes (Figure 8B, middle panel). It remains unclear why caveolin-1 localization peaked in fraction 2, while TRPC6 and SNF8 in the low density fractions were mainly in fraction 3, but the generation of distinct lipid raft subfractions has been reported using a protocol similar to the one used here [56].

\section{Discussion}

Here we identify SNF8 as a novel binding partner of TRPC6. The interaction was first established in a yeast two-hybrid screen, where it was found to be mediated by the amino terminal 107 amino acids of TRPC6. The TRPC6-SNF8 interaction has been confirmed by coimmunoprecipitation, and the proteins partially colocalize in cells. Importantly, SNF8 overexpression enhances whole cell current amplitudes mediated by both wildtype and FSGS-associated mutant TRPC6, as well as mutant TRPC6-mediated NFAT activation, while RNAi mediated knock-down of endogenous SNF8 diminishes NFAT activation by mutant TRPC6. The effect of SNF8 on TRPC6 channel activity and downstream activation of NFAT-mediated transcription does not appear to be mediated by alterations in the global surface expression levels of TRPC6 or a shift of channel into lipid rafts. Taken together, these results suggest a novel role for SNF8 in the modulation of TRPC6 channel activity.

It is difficult to reconcile the ability of SNF8 to enhance TRPC6-mediated current with its major known function, sorting transmembrane proteins into multivesiculated bodies for degradation. SNF8, together with VPS25 and VPS36, forms the endosomal sorting complex for transport (ESCRT) -II complex [35]. ESCRT-II, in conjunction with the ESCRT-I and -III complexes, is involved in the budding of intraluminal vesicles from endosomes to form multivesiculated bodies (MVB) (reviewed in $[37,39,40]$ ). This process is critical for the downregulation of multiple signaling receptors, including the yeast mating receptor Ste2 [35], EGFR[36,57] and Notch $[58,59]$. The ESCRT complexes not only target ubiquitinated transmembrane proteins to the vesicles 
A

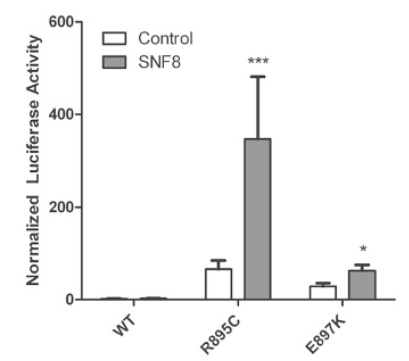

C

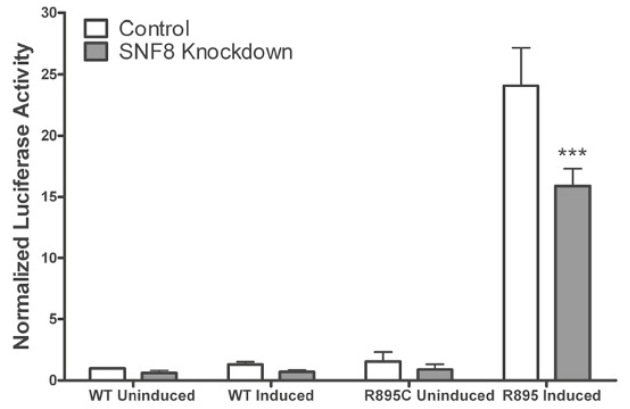

B

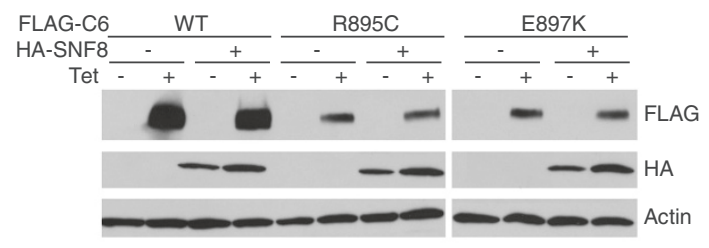

D

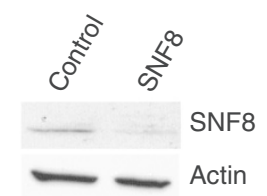

Figure 6 SNF8 effect on NFAT-mediated transcription. A. Cells stably expressing tetracycline-inducible wild-type, R895C or E897K TRPC6 were transfected with an NFAT-responsive luciferase reporter plasmid and either control plasmid or HA-SNF8 expression plasmid. Normalized luciferase activity was determined after TRPC6 expression was induced for 24 hours. Results are means \pm SEM; one way ANOVA; * p $<0.05$ vs. control; ${ }^{* * *} p<0.001$ vs. control. B. Western blots to confirm the expression of FLAG-TRPC6 and HA-SNF8 in lysates from cells used in the luciferase assays in (A). Actin was utilized as a loading control. C. Cells expressing wild-type or R895C TRPC6 under a tetracycline inducible promoter were transfected with plasmid encoding for either SNF8 shRNA (SNF8 Knockdown) or a scrambled shRNA (control), and luciferase reporter plasmids. 24 hours after transfection, TRPC6 expression was induced by the addition of tetracycline as indicated (induced). Cells cultured in the absence of tetracycline (uninduced) did not express detectable amounts of TRPC6, and were used as controls. 24 hours post-induction, luciferase reporter assays were performed. Results are means $\pm \mathrm{SEM}$; one way ANOVA, ${ }^{* *} \mathrm{p}<0.001$ vs control shRNA. D. M1R cells were transfected with plasmids encoding for either an shRNA targeting SNF8, or a scrambled shRNA (control), as well as GFP. 48 hours post-transfection, cells were trypsinized and GFP expressing cells isolated by FACS cell sorting. Lysates from GFP positive cells were analyzed by Western blot for expression of SNF8. Actin expression was used as a loading control.

of the MVB, but induce the necessary membrane curvature and ultimate scission of the vesicles from their parent membrane [60-62]. In addition to their role in MVB formation, ESCRTs are involved in the membrane

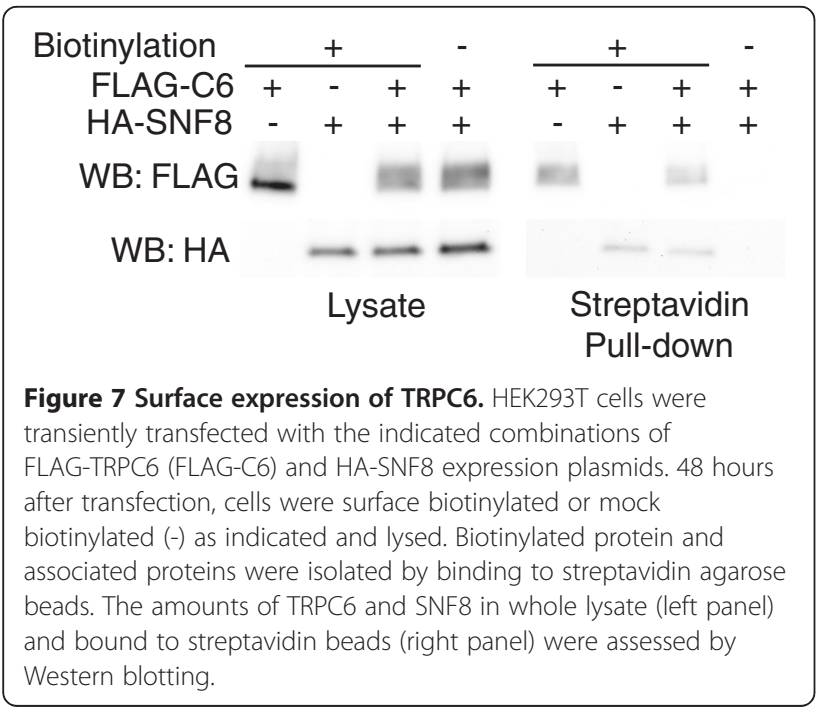

scission required for cytokinesis and the budding of enveloped viruses $[63,64]$.

Whether the endosomal-lysosomal functions of SNF8 might relate to TRPC6 activity is unclear. It is worth noting that SNF8 has been implicated to have several non-endosomal functions. These include the establishment of the bicoid mRNA gradient in the Drosophila oocyte [65] and modulation of the ELL transcription elongation complex [66]. Perhaps most relevant to its role in enhancing TRPC6-mediated currents, the yeast homologue of SNF8, VPS22, as well as other members of the ESCRT complexes, have been shown to be involved in the trafficking and surface expression of the sodium pump Ena1 [43]. Although we have not been able to detect a change in the amount of total TRPC6 expressed on the cell surface or in lipid rafts in response to co-expression of SNF8, it is possible that SNF8 traffics the channel to a subdomain of the plasma membrane where TRPC6 activity is enhanced. Along these lines, it is noteworthy that podocin enhances TRPC6 activity in a cholesterol dependent manner without altering plasma membrane expression [14], while the differential 


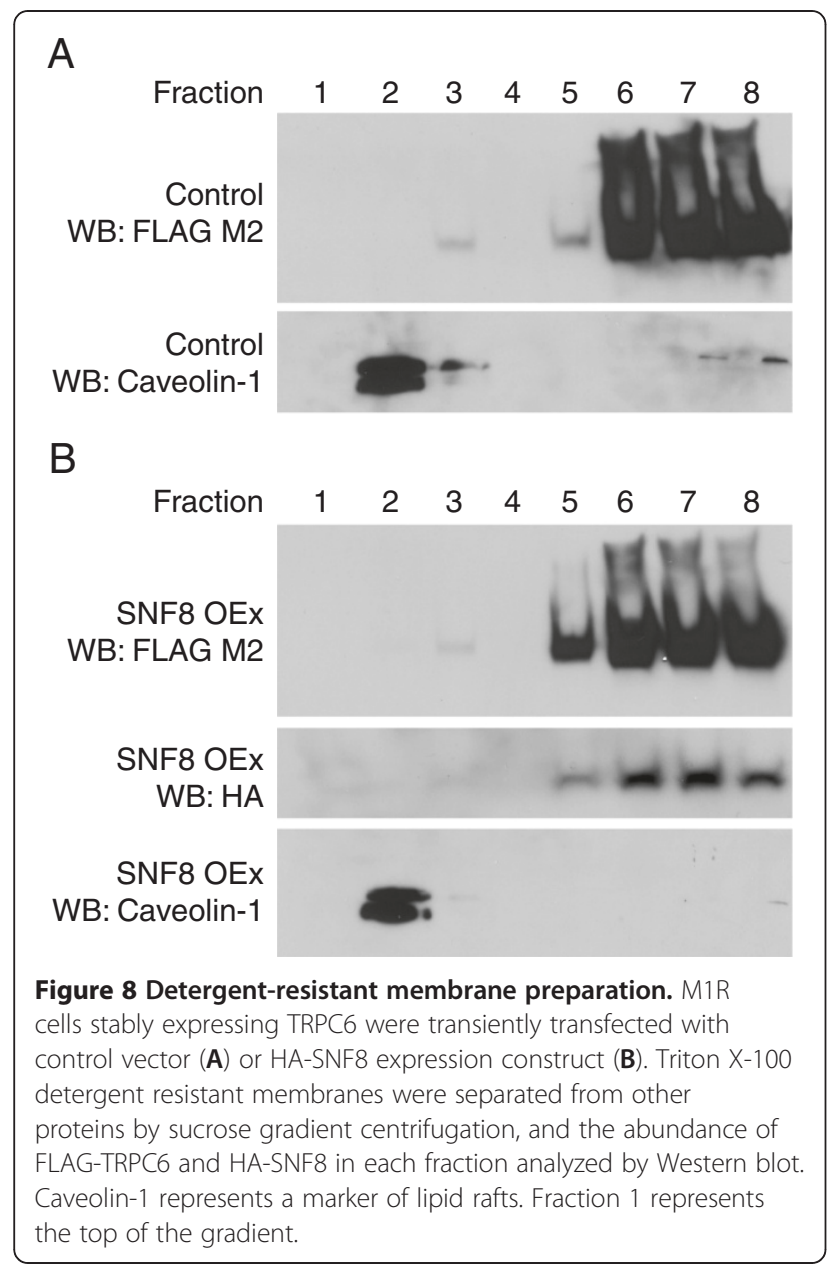

requirement of ESCRT-II for the budding of avian sarcoma and leukosis virus (ASLV) and human immunodeficiency virus, type-1 (HIV-1), correlates with their assembly on phosphatidylethanolamine (PE) containing or PE-negative membranes, respectively $[63,67]$. Alternatively, it is possible that SNF8 regulates TRPC6 indirectly by competing with a negative regulator or by altering the activity of another membrane protein in the vicinity of TRPC6, such as an enzyme that affects phosphoinositide levels. Finally, TRPC6 has been reported to be activated by membrane deformation [68], though this has been disputed [69]. One could hypothesize that SNF8 may act to recruit ESCRT-II to TRPC6, and alter TRPC6 function through local convex deformation of the membrane [70]. Further understanding the mechanism whereby SNF8 enhances TRPC6 currents will be the goal of future investigations.

\section{Conclusion}

This work shows that: 1 . SNF8 is a potential binding partner of TRPC6, 2. overexpression of SNF8 enhances both wild-type and mutant TRPC6 current densities, and 3. modulating SNF8 expression levels affects NFAT activation downstream of gain-of-function, FSGSassociated TRPC6 mutations. The mechanism for regulating channel activity is not mediated by changes in global cell surface expression or recruitment into lipid rafts. Taken together, these results identify SNF8 as a potent modulator of the TRPC6 channel.

\section{Methods}

\section{Plasmids and reagents}

The human TRPC6 coding sequence, with or without mutations as outlined in the text, and containing an amino-terminal FLAG tag sequence, was cloned into pcDNA4/TO/myc-HIS B (Clontech) using standard PCR-based techniques. Similarly, full-length human TRPC6 carrying an amino-terminal HA tag was amplified by PCR and subcloned into pcDNA3.1. The HA-SNF8 expression plasmid was a gift from C. Bucci [71]. The Matchmaker Two-Hybrid System and S. cerevisiae Y187 pre-transformed with a human kidney cDNA library were purchased from Clontech (Palo Alto, CA). The dual luciferase assay kit and reporter vectors pGL4.30 and pGL4.74 were obtained from Promega. Affinity purified rabbit anti-TRPC6 polyclonal antibody was purchased from Chemicon, anti-FLAG M2 monoclonal antibody and anti-FLAG rabbit polyclonal antibodies were purchased from Sigma, rabbit anti-GFP polyclonal antibody and mouse anti-HA monoclonal antibody were purchased from Abcam Inc, and rabbit anti-HA monoclonal antibody (C29F4) was purchased from Cell Signaling Technologies. Anti-SNF8 rabbit polyclonal antibody was the kind gift of Dr. H. Stenmark [36]. Anti-caveolin-1 mouse monoclonal antibody (clone 2297) was obtained from BD Biosciences.

\section{Yeast two-hybrid screen}

cDNA encoding residues 1 through 406 of TRPC6 (wildtype N-terminal domain) was used as bait and cloned in-frame with GAL4 DNA-binding domain in the vector pGBKT7-BD and transformed into yeast strain AH109. The bait strain was mated to Y187 yeast strain pretransformed with a commercially available human kidney cDNA library cloned into pACT2-AD vector according to the manufacturer's protocol (Clontech). Based on mating efficiency, $1 \times 10^{6}$ clones were screened. Mated yeast cells were grown on highstringency selection plates (SD-Leu, Trp, Ade, His) and positive colonies were further verified by growth on high-stringency plates supplemented with $\mathrm{X}$ - $\alpha$-gal as a test for $\beta$-galactosidase activity. pACT2-AD plasmids containing library inserts from positive colonies were isolated and transformed into $\mathrm{DH} 5 \alpha$ derived E. coli (New England Biolabs). Plasmids were then isolated 
from bacteria, sequenced, and analyzed using the BlastNT alignment algorithm from NCBI.

To confirm the interaction, the full-length SNF8 coding sequence was cloned inframe into the pGAD-T7 plasmid and the resulting plasmid transformed into Y187 strain yeast. The resulting strain was mated with AH109 bait strain harboring pGBKT7 with wild-type TRPC6 N-terminal sequences or the TRPC6 C-terminal domain (corresponding to amino acids 726-931). The resulting diploids were tested for positive interaction by growth on high-stringency plates.

\section{Cell culture and luciferase assays}

Cells stably expressing the M1 muscarinic acetylcholine receptor and either wild-type, R895C or E897K TRPC6 under a tetracycline-inducible promoter were generated from T-Rex-293 cells (Invitrogen), as previously described [38]. Stable cell lines were induced to express TRPC6 for 24 hours prior to lysis by adding tetracycline to a $1 \mu \mathrm{g} / \mathrm{ml}$ final concentration in culture medium. Luciferase assays were carried out essentially as previously described [38] using the dual luciferase reporter assay (DLR; Roche) using a Veritas microplate luminometer (Turner Biosystems). All conditions were tested in triplicate and results normalized to the Renilla luciferase internal control.

\section{RNA interference}

SNF8 was knocked down using short hairpin-expressing double-stranded oligonucleotides cloned into pcDNA 6.2-GW/EmGFP-miR plasmids (BLOCK-iT ${ }^{\mathrm{TM}}$ Pol II miR RNAi Expression Vector Kit, Invitrogen). A specific shRNA sequence against SNF8 (sense 5'-TGACTTCG [CC]CAATGTCAGTCAA- $3^{\prime}$ and antisense $5^{\prime}$-TTGACT GACATCCTGGGCGAA-3'), and a scrabbled control sequence, were obtained from Invitrogen and cloned into the plasmid. Knock-down efficiency was determined by transient transfection of M1R cells with either SNF8 or control plasmid, followed by FACS sorting for GFP expression to isolate the transfected cells after 48 hrs. SNF8 expression in sorted cells was assessed by immunbloting for SNF8.

To assess the effect of SNF8 knock-down on NFATmediated transcription, cells were transfected with the indicated shRNA expression vector and luciferase reporter plasmids using Fugene 6. 24 hours after transfection, TRPC6 expression was induced where indicated by the addition of tetracycline $(1 \mu \mathrm{g} / \mathrm{ml}$ final concentration) to the media. After an additional 24 hours, cells were lysed and processed for dual luciferase reporter assay.

\section{Immunoprecipitation and Immunoblotting}

Transfected cells were rinsed once in phosphate buffered saline and lysed in modified RIPA lysis buffer $(50 \mathrm{mM}$
Tris, $\mathrm{pH}$ 8.0, $150 \mathrm{mM} \mathrm{NaCl}, 1 \% \mathrm{NP}-40,0.5 \%$ sodium deoxycholate supplemented with Complete protease inhibitor cocktail (Roche)). After clearing lysates by centrifugation at $14,000 \mathrm{RPM}$ for 15 minutes at $4^{\circ} \mathrm{C}$, supernatants were incubated with $30 \mu \mathrm{l}$ of FLAG M2 agarose slurry (Sigma-Aldrich), and incubated with constant agitation at $4^{\circ} \mathrm{C}$ for 2-3 hours. Immunoprecipitated complexes were washed three times with lysis buffer and eluted off of the beads by boiling in SDS-sample loading buffer.

Cell lysates and immunoprecipitated materials were separated by SDS-PAGE and transferred to PVDF membrane (Bio-Rad). The membrane was blocked with 5\% nonfat milk in PBST (PBS with 0.05\% Tween-20) for 1 hour at room temperature, followed by overnight incubation in 1:1000 anti-TRPC6, 1:500 anti-HA, 1:500 FLAG M2, or 1:200 anti-SNF8 antibody in 5\% non-fat milk PBST. After three washes in PBST, blots were incubated with the appropriate secondary antibody conjugated to HRP (Pierce) in PBST at room temperature, followed by detection with SuperSignal West Pico chemiluminescent substrate (Pierce).

\section{Fluorescence microscopy}

Cells stably expressing wild-type TRPC6 under a tetracycline inducible promoter were grown on collagen I coated glass coverslips and transfected with plasmid encoding for HA-SNF8 using Fugene6 followed by treatment with tetracycline to induce FLAG-TRPC6. 24 hours after inducing TRPC6 expression, cells were washed with PBS, fixed in $2 \%$ paraformaldehyde, $4 \%$ sucrose in PBS, and permeabilized with $0.3 \%$ Triton X-100 in PBS. Nonspecific binding sites were blocked using blocking solution ( $2 \%$ bovine serum albumin, $2 \%$ fetal calf serum, $0.2 \%$ fish gelatin). Cells were incubated with 1:200 anti-FLAG M2 antibody and 1:1600 anti-HA rabbit monoclonal antibody, followed by Alexa488 conjugated goat anti-mouse and Cy3 conjugated goat antirabbit secondary antibody (Jackson ImmunoResearch), and mounted on slides with ProLong Gold antifade reagent (Invitrogen). Images were taken using a Zeiss LSM 510 confocal microscope.

\section{Electrophysiology}

Patch-clamp electrophysiology (Axopatch 200B amplifier, Axon Instruments, $\mathrm{CA}$ ) was performed in the wholecell configuration. Briefly, HEK293T cells (American Type Culture Collection, VA) were plated on glass coverslips at low density and placed in the recording chamber. The patch pipettes with resistances of 3-4 $\mathrm{M} \Omega$ were pulled from borosilicate glass with a P97 puller (Sutter Instrument) and filled with a solution containing (in mM): $135 \mathrm{CH} 3 \mathrm{SO} 3 \mathrm{Cs}, 10 \mathrm{CsCl}, 3 \mathrm{MgATP}$, 0.2 NaGTP, 0.2 EGTA, $0.13 \mathrm{CaCl}$, and 10 HEPES, 
$\mathrm{pH} 7.3$ with $\mathrm{CsOH}$. The bath solution contained (in $\mathrm{mM}): 135 \mathrm{CH} 3 \mathrm{SO} 3 \mathrm{Na}, 5 \mathrm{CsCl}, 2 \mathrm{CaCl} 2,1 \mathrm{MgCl} 2,10$ HEPES, and 10 Glucose, pH 7.4 with $\mathrm{NaOH}$. Carbachol $(100 \mu \mathrm{M})$ or 1-oleoyl-2-acetyl-sn-glycerol (OAG, $10 \mu \mathrm{M})$ was applied to the bath solution. Whole-cell currents were recorded from $-100 \mathrm{mV}$ to $100 \mathrm{mV}$ voltage ramps over $150 \mathrm{~ms}$ and a holding potential of $0 \mathrm{mV}$. All data were acquired at room temperature and analyzed using pClamp 10 (Axon Instruments, CA). Statistical analysis was done using one-way ANOVA Fisher's LSD test, and p values $<0.05$ were considered significant.

\section{Surface biotinylation}

Cells were surface biotinylated on ice using Sulfo-NHSSS-biotin (Pierce) 48 hours after transfection, essentially as previously described [38], followed by lysis in modified RIPA buffer. Lysates were incubated with streptavidin beads (Pierce) at $4^{\circ} \mathrm{C}$. The streptavidin beads were extensively washed and bound material was eluted and analyzed by SDS-PAGE electrophoresis followed by Western blot analysis.

\section{Lipid raft preparation}

M1R cells stably expressing FLAG-tagged TRPC6 were transfected with control or HA-SNF8 expression constructs. 48 hours after transfection, cells were processed using a protocol modified from Alicia et al [54]. Briefly, cells were washed in ice cold PBS, scraped off of plates in PBS and lysed in 1\% Triton X-100 in MES buffer (25mM MES, pH 6.5, 150mM NaCl, 2mM EDTA complemented with protease inhibitors) on ice for 20 minutes. Lysates were further homogenized in a glass homogenizer with the loose fitting piston, then mixed with an equal volume of $85 \%(\mathrm{w} / \mathrm{v})$ sucrose in MES buffer. Lysates were overlayed with $4 \mathrm{mls}$ of $40 \%$ sucrose in MES buffer followed by $4 \mathrm{mls}$ of $5 \%$ sucrose in MES buffer. Lysates were spun at $175,000 \mathrm{x}$ g for 24 hours in an SW41 Ti rotor. Eight $1.5 \mathrm{ml}$ fractions were obtained by aspiration starting at the top of the gradient. Aliqouts of each fraction were combined with $4 \mathrm{x}$ sample loading buffer with $\beta$-mercaptoethanol and analyzed by Western blot as above.

\section{Abbreviations}

ASLV: Avian sarcoma and leukosis virus; ESCRT: Endosomal sorting complex for transport; FSGS: Focal segmental glomerulosclerosis; GFP: Green fluorescent protein; HA: Hemaglutinin; HIV: Human immunodeficiency virus; NFAT: Nuclear factor of activated T-cells; PAGE: Poly-accrylamide gel electrophoresis, PE, Phosphatidylethanolamine; shRNA: Short hairpin ribonucleic acid; TRPC: Canonical transient receptor potential.
}

\section{Competing interests}

The authors declare that they have no competing interest.

\section{Authors' contributions}

$\mathrm{RC}$ performed the yeast-two hybrid screen and candidate identification, generated shRNA plasmids, performed SNF8 knockdown experiments and luciferase assays, and helped draft the manuscript. SK generated expression constructs, and performed cell transfections and immunoblotting. MRP participated in the design and coordination of the study. DT and AG performed all electrophysiology experiments and related statistical analysis, and helped draft the manuscript. JS conceived of the study, performed co-immunoprecipitation, immunofluorescence, surface biotinylation, membrane fractionation and luciferase assays, performed statistical analysis and drafted the manuscript. All authors read and approved the final manuscript.

\section{Acknowledgements}

This work was supported by NIH grants DK743082 and DK080947 and an ASN Young Investigator Award (to J.S.), by NIH grant DK59588 (to M.R.P.), a Graduate Prize Fellowship from the Harvard University Graduate School of Arts and Sciences and a grant from the Office of Enrichment Programs at Harvard Medical School (to R.C.), and by a Nephcure Young Investigator Award, an ASN Young Investigator Award and NIH grant DK083511 (to A.G.). We are grateful to Dr. Cecilia Bucci for the HA-SNF8 expression construct and to Dr. Harald Stenmark for the kind gift of anti-SNF8 antibody. We would like to thank Arnolt Ramos for assistance with electrophysiology experiments. We appreciate the assistance of the Beth Israel Deaconess Confocal Imaging Core and the Dana Farber Cancer Center Flow Cytometry Core facilities. We thank the members of the Pollak lab for helpful discussions and suggestions.

\section{Author details}

1 Division of Nephrology, Beth Israel Deaconess Medical Center, Research North 304B, 99 Brookline Ave, Boston, MA 02215, USA. ²Department of Medicine, Nephrology Division, Massachusetts General Hospital, 149 13th Street, Room 8.102, Boston, MA 02114, USA. ${ }^{3}$ Harvard Medical School, Boston, MA 02115, USA.

Received: 10 July 2012 Accepted: 23 October 2012

Published: 21 November 2012

\section{References}

1. Montell C: The TRP superfamily of cation channels. Sci STKE 2005, 2005(272):re3.

2. Hofmann T, Schaefer M, Schultz G, Gudermann T: Subunit composition of mammalian transient receptor potential channels in living cells. Proc Natl Acad Sci U S A 2002, 99(11):7461-7466.

3. Vazquez G, Wedel BJ, Aziz O, Trebak M, Putney JW Jr: The mammalian TRPC cation channels. Biochim Biophys Acta 2004, 1742(1-3):21-36.

4. Kim EY, Alvarez-Baron CP, Dryer SE: Canonical transient receptor potential channel (TRPC)3 and TRPC6 associate with large-conductance $\mathrm{Ca} 2+$-activated $\mathrm{K}+(\mathrm{BKCa})$ channels: role in BKCa trafficking to the surface of cultured podocytes. Mol Pharmacol 2009, 75(3):466-477.

5. Boulay $\mathrm{G}: \mathrm{Ca}(2+)$-calmodulin regulates receptor-operated $\mathrm{Ca}(2+)$ entry activity of TRPC6 in HEK-293 cells. Cell Calcium 2002, 32(4):201-207.

6. Kwon Y, Hofmann T, Montell C: Integration of phosphoinositide- and calmodulin-mediated regulation of TRPC6. Mol Cell 2007, 25(4):491-503.

7. Tang J, Lin Y, Zhang Z, Tikunova S, Birnbaumer L, Zhu MX: Identification of common binding sites for calmodulin and inositol 1,4,5-trisphosphate receptors on the carboxyl termini of trp channels. J Biol Chem 2001, 276(24):21303-21310.

8. Sinkins WG, Goel M, Estacion M, Schilling WP: Association of immunophilins with mammalian TRPC channels. J Biol Chem 2004, 279(33):34521-34529.

9. Hisatsune $C$, Kuroda Y, Nakamura K, Inoue T, Nakamura T, Michikawa T, Mizutani A, Mikoshiba K: Regulation of TRPC6 channel activity by tyrosine phosphorylation. J Biol Chem 2004, 279(18):18887-18894.

10. Boulay G, Brown DM, Qin N, Jiang M, Dietrich A, Zhu MX, Chen Z, Birnbaumer M, Mikoshiba K, Birnbaumer L: Modulation of $\mathrm{Ca}(2+)$ entry by polypeptides of the inositol 1,4, 5-trisphosphate receptor (IP3R) that bind transient receptor potential (TRP): evidence for roles of TRP and IP3R in store depletion-activated $\mathrm{Ca}(2+)$ entry. Proc Natl Acad Sci U S A 1999, 96(26):14955-14960.

11. Lussier MP, Cayouette S, Lepage PK, Bernier CL, Francoeur N, St-Hilaire M, Pinard M, Boulay G: MxA, a member of the dynamin superfamily, interacts with the ankyrin-like repeat domain of TRPC. J Biol Chem 2005, 280(19):19393-19400. 
12. Goel M, Sinkins W, Keightley A, Kinter M, Schilling WP: Proteomic analysis of TRPC5- and TRPC6-binding partners reveals interaction with the plasmalemmal $\mathrm{Na}(+) / \mathrm{K}(+)$-ATPase. Pflugers Arch 2005, 451(1):87-98.

13. Kanda S, Harita Y, Shibagaki $Y$, Sekine T, Igarashi T, Inoue T, Hattori S: Tyrosine phosphorylation-dependent activation of TRPC 6 regulated by PLC-gamma1 and nephrin: effect of mutations associated with focal segmental glomerulosclerosis. Mol Biol Cell 2011, 22(11):1824-1835.

14. Huber TB, Schermer B, Muller RU, Hohne M, Bartram M, Calixto A, Hagmann H, Reinhardt C, Koos F, Kunzelmann K, et al: Podocin and MEC-2 bind cholesterol to regulate the activity of associated ion channels. Proc Natl Acad Sci U S A 2006, 103(46):17079-17086.

15. Reiser J, Polu KR, Moller CC, Kenlan P, Altintas MM, Wei C, Faul C, Herbert S, Villegas I, Avila-Casado C, et al: TRPC6 is a glomerular slit diaphragmassociated channel required for normal renal function. Nat Genet 2005, 37(7):739-744

16. Kini $V$, Chavez $A$, Mehta D: A new role for PTEN in regulating transient receptor potential canonical channel 6-mediated Ca2+ entry, endothelial permeability, and angiogenesis. J Biol Chem 2010, 285(43):33082-33091.

17. Lussier MP, Lepage PK, Bousquet SM, Boulay G: RNF24, a new TRPC interacting protein, causes the intracellular retention of TRPC. Cell Calcium 2008, 43(5):432-443.

18. Shi J, Mori E, Mori Y, Mori M, Li J, Ito Y, Inoue R: Multiple regulation by calcium of murine homologues of transient receptor potential proteins TRPC6 and TRPC7 expressed in HEK293 cells. J Physio/ 2004, 561(Pt 2):415-432.

19. Kim JY, Saffen D: Activation of M1 muscarinic acetylcholine receptors stimulates the formation of a multiprotein complex centered on TRPC6 channels. J Biol Chem 2005, 280(36):32035-32047.

20. Takahashi S, Lin H, Geshi N, Mori Y, Kawarabayashi Y, Takami N, Mori MX, Honda A, Inoue R: Nitric oxide-cGMP-protein kinase G pathway negatively regulates vascular transient receptor potential channel TRPC6. J Physiol 2008, 586(Pt 17):4209-4223.

21. Bousquet SM, Monet M, Boulay G: Protein kinase C-dependent phosphorylation of transient receptor potential canonical 6 (TRPC6) on serine 448 causes channel inhibition. J Biol Chem 2010, 285(52):40534-40543.

22. Lemonnier L, Trebak M, Putney JW Jr: Complex regulation of the TRPC3, 6 and 7 channel subfamily by diacylglycerol and phosphatidylinositol-4,5-bisphosphate. Cell Calcium 2008, 43(5):506-514.

23. Monet M, Francoeur N, Boulay G: Involvement of Phosphoinositide 3-Kinase and PTEN Protein in Mechanism of Activation of TRPC6 Protein in Vascular Smooth Muscle Cells. J Biol Chem 2012, 287(21):17672-17681.

24. Winn MP, Conlon PJ, Lynn KL, Farrington MK, Creazzo T, Hawkins AF, Daskalakis N, Kwan SY, Ebersviller S, Burchette JL, et al: A mutation in the TRPC6 cation channel causes familial focal segmental glomerulosclerosis. Science 2005, 308(5729):1801-1804.

25. Moller CC, Wei C, Altintas MM, Li J, Greka A, Ohse T, Pippin JW, Rastaldi MP, Wawersik S, Schiavi S, et al: Induction of TRPC6 channel in acquired forms of proteinuric kidney disease. J Am Soc Nephrol 2007, 18(1):29-36.

26. Wang Z, Wei X, Zhang Y, Ma X, Li B, Zhang S, Du P, Zhang X, Yi F: NADPH oxidase-derived ROS contributes to upregulation of TRPC6 expression in puromycin aminonucleoside-induced podocyte injury. Cell Physiol Biochem 2009, 24(5-6):619-626.

27. Eckel J, Lavin PJ, Finch EA, Mukerji N, Burch J, Gbadegesin R, Wu G, Bowling B, Byrd A, Hall G, et al: TRPC6 enhances angiotensin II-induced albuminuria. J Am Soc Nephrol 2011, 22(3):526-535.

28. Heeringa SF, Moller CC, Du J, Yue L, Hinkes B, Chernin G, Vlangos CN, Hoyer PF, Reiser J, Hildebrandt F: A novel TRPC6 mutation that causes childhood FSGS. PLOS One 2009, 4(11):e7771.

29. Zhu B, Chen N, Wang ZH, Pan XX, Ren H, Zhang W, Wang WM: Identification and functional analysis of a novel TRPC6 mutation associated with late onset familial focal segmental glomerulosclerosis in Chinese patients. Mutat Res 2009, 664(1-2):84-90.

30. Onohara $N$, Nishida M, Inoue R, Kobayashi H, Sumimoto $H$, Sato $Y$, Mori $Y$, Nagao T, Kurose H: TRPC3 and TRPC6 are essential for angiotensin IIinduced cardiac hypertrophy. EMBO J 2006, 25(22):5305-5316.

31. Kuwahara K, Wang Y, McAnally J, Richardson JA, Bassel-Duby R, Hill JA Olson EN: TRPC6 fulfills a calcineurin signaling circuit during pathologic cardiac remodeling. J Clin Invest 2006, 116(12):3114-3126.

32. Weissmann N, Dietrich A, Fuchs B, Kalwa H, Ay M, Dumitrascu R, Olschewski A, Storch U, Mederos y Schnitzler M, Ghofrani HA, et al: Classical transient receptor potential channel 6 (TRPC6) is essential for hypoxic pulmonary vasoconstriction and alveolar gas exchange. Proc Natl Acad Sci U S A 2006, 103(50):19093-19098.

33. Weissmann N, Sydykov A, Kalwa H, Storch U, Fuchs B, Mederos y Schnitzler M, Brandes RP, Grimminger $F$, Meissner $M$, Freichel $M$, et al: Activation of TRPC6 channels is essential for lung ischaemia-reperfusion induced oedema in mice. Nat Commun 2012, 3:649.

34. Paez Espinosa EV, Murad JP, Ting HJ, Khasawneh FT: Mouse transient receptor potential channel 6: role in hemostasis and thrombogenesis. Biochem Biophys Res Commun 2012, 417(2):853-856.

35. Babst M, Katzmann DJ, Snyder WB, Wendland B, Emr SD: Endosomeassociated complex, ESCRT-II, recruits transport machinery for protein sorting at the multivesicular body. Dev Cell 2002, 3(2):283-289.

36. Malerod L, Stuffers S, Brech A, Stenmark H: Vps22/EAP30 in ESCRT-II mediates endosomal sorting of growth factor and chemokine receptors destined for lysosomal degradation. Traffic 2007, 8(11):1617-1629.

37. Raiborg C, Stenmark H: The ESCRT machinery in endosomal sorting of ubiquitylated membrane proteins. Nature 2009, 458(7237):445-452.

38. Schlondorff J, Del Camino D, Carrasquillo R, Lacey V, Pollak MR: TRPC6 mutations associated with focal segmental glomerulosclerosis cause constitutive activation of NFAT-dependent transcription. Am J Physiol Cell Physiol 2009, 296(3):C558-C569.

39. Hanson PI, Shim S, Merrill SA: Cell biology of the ESCRT machinery. Curr Opin Cell Biol 2009, 21(4):568-574.

40. Wollert T, Yang D, Ren X, Lee HH, Im YJ, Hurley JH: The ESCRT machinery at a glance. J Cell Sci 2009, 122(Pt 13):2163-2166.

41. Bezzerides VJ, Ramsey IS, Kotecha S, Greka A, Clapham DE: Rapid vesicular translocation and insertion of TRP channels. Nat Cell Biol 2004, 6(8):709-720.

42. Cayouette S, Lussier MP, Mathieu EL, Bousquet SM, Boulay G: Exocytotic insertion of TRPC6 channel into the plasma membrane upon $\mathrm{Gq}$ protein-coupled receptor activation. J Biol Chem 2004, 279(8):7241-7246

43. Logg K, Warringer J, Hashemi SH, Kall M, Blomberg A: The sodium pump Ena1p provides mechanistic insight into the salt sensitivity of vacuolar protein sorting mutants. Biochim Biophys Acta 2008, 1783(6):974-984.

44. Aires $V$, Hichami A, Boulay G, Khan NA: Activation of TRPC6 calcium channels by diacylglycerol (DAG)-containing arachidonic acid: a comparative study with DAG-containing docosahexaenoic acid. Biochimie 2007, 89(8):926-937.

45. Brazer SC, Singh BB, Liu X, Swaim W, Ambudkar IS: Caveolin-1 contributes to assembly of store-operated $\mathrm{Ca} 2+$ influx channels by regulating plasma membrane localization of TRPC1. J Biol Chem 2003, 278(29):27208-27215.

46. Brownlow SL, Sage SO: Transient receptor potential protein subunit assembly and membrane distribution in human platelets. Thromb Haemost 2005, 94(4):839-845.

47. Kwiatek AM, Minshall RD, Cool DR, Skidgel RA, Malik AB, Tiruppathi C: Caveolin-1 regulates store-operated $\mathrm{Ca} 2+$ influx by binding of its scaffolding domain to transient receptor potential channel-1 in endothelial cells. Mol Pharmacol 2006, 70(4):1174-1183.

48. Lockwich TP, Liu X, Singh BB, Jadlowiec J, Weiland S, Ambudkar IS: Assembly of Trp1 in a signaling complex associated with caveolinscaffolding lipid raft domains. J Biol Chem 2000, 275(16):11934-11942.

49. Murata T, Lin MI, Stan RV, Bauer PM, Yu J, Sessa WC: Genetic evidence supporting caveolae microdomain regulation of calcium entry in endothelial cells. J Biol Chem 2007, 282(22):16631-16643.

50. Pani B, Singh BB: Lipid rafts/caveolae as microdomains of calcium signaling. Cell Calcium 2009, 45(6):625-633.

51. Jardin I, Salido GM, Rosado JA: Role of lipid rafts in the interaction between hTRPC1, Orai1 and STIM1. Channels (Austin) 2008, 2(6):401-403.

52. Pani B, Ong HL, Liu X, Rauser K, Ambudkar IS, Singh BB: Lipid rafts determine clustering of STIM1 in endoplasmic reticulum-plasma membrane junctions and regulation of store-operated Ca2+ entry (SOCE). J Biol Chem 2008, 283(25):17333-17340.

53. Sundivakkam PC, Kwiatek AM, Sharma TT, Minshall RD, Malik AB, Tiruppathi C: Caveolin-1 scaffold domain interacts with TRPC1 and IP3R3 to regulate $\mathrm{Ca} 2+$ store release-induced $\mathrm{Ca} 2+$ entry in endothelial cells. Am J Physiol Cell Physiol 2009, 296(3):C403-C413.

54. Alicia S, Angelica Z, Carlos S, Alfonso S, Vaca L: STIM1 converts TRPC1 from a receptor-operated to a store-operated channel: moving TRPC1 in and out of lipid rafts. Cell Calcium 2008, 44(5):479-491. 
55. Pani B, Ong HL, Brazer SC, Liu X, Rauser K, Singh BB, Ambudkar IS: Activation of TRPC1 by STIM1 in ER-PM microdomains involves release of the channel from its scaffold caveolin-1. Proc Natl Acad Sci U S A 2009, 106(47):20087-20092.

56. Mellgren RL: Detergent-resistant membrane subfractions containing proteins of plasma membrane, mitochondrial, and internal membrane origins. J Biochem Biophys Methods 2008, 70(6):1029-1036.

57. Langelier C, von Schwedler UK, Fisher RD, De Domenico I, White PL, Hill CP, Kaplan J, Ward D, Sundquist WI: Human ESCRT-II complex and its role in human immunodeficiency virus type 1 release. J Virol 2006, 80(19):9465-9480.

58. Herz HM, Woodfield SE, Chen Z, Bolduc C, Bergmann A: Common and distinct genetic properties of ESCRT-II components in Drosophila. PLoS One 2009, 4(1):e4165.

59. Vaccari T, Rusten TE, Menut L, Nezis IP, Brech A, Stenmark H, Bilder D: Comparative analysis of ESCRT-I, ESCRT-II and ESCRT-III function in Drosophila by efficient isolation of ESCRT mutants. J Cell Sci 2009, 122(Pt 14):2413-2423.

60. Hanson Pl, Roth R, Lin Y, Heuser JE: Plasma membrane deformation by circular arrays of ESCRT-III protein filaments. J Cell Biol 2008, 180(2):389-402.

61. Im YJ, Wollert T, Boura E, Hurley JH: Structure and function of the ESCRT-II-III interface in multivesicular body biogenesis. Dev Cell 2009, 17(2):234-243.

62. Saksena S, Wahlman J, Teis D, Johnson AE, Emr SD: Functional reconstitution of ESCRT-III assembly and disassembly. Cell 2009, 136(1):97-109.

63. Pincetic A, Leis J: The Mechanism of Budding of Retroviruses From Cell Membranes. Adv Virol 2009, 2009:6239691-6239699.

64. McDonald B, Martin-Serrano J: No strings attached: the ESCRT machinery in viral budding and cytokinesis. J Cell Sci 2009, 122(Pt 13):2167-2177.

65. Irion $U$, St Johnston D: Bicoid RNA localization requires specific binding of an endosomal sorting complex. Nature 2007, 445(7127):554-558.

66. Schmidt AE, Miller T, Schmidt SL, Shiekhattar R, Shilatifard A: Cloning and characterization of the EAP30 subunit of the ELL complex that confers derepression of transcription by RNA polymerase II. J Biol Chem 1999, 274(31):21981-21985.

67. Medina G, Pincetic A, Ehrlich LS, Zhang Y, Tang Y, Leis J, Carter CA: Tsg101 can replace Nedd4 function in ASV Gag release but not membrane targeting. Virology 2008, 377(1):30-38.

68. Spassova MA, Hewavitharana T, Xu W, Soboloff J, Gill DL: A common mechanism underlies stretch activation and receptor activation of TRPC6 channels. Proc Natl Acad Sci U S A 2006, 103(44):16586-16591.

69. Gottlieb P, Folgering J, Maroto R, Raso A, Wood TG, Kurosky A, Bowman C, Bichet D, Patel A, Sachs F, et al: Revisiting TRPC1 and TRPC6 mechanosensitivity. Pflugers Arch 2008, 455(6):1097-1103.

70. Wollert T, Hurley JH: Molecular mechanism of multivesicular body biogenesis by ESCRT complexes. Nature 2010, 464(7290):864-869.

71. Progida C, Spinosa MR, De Luca A, Bucci C: RILP interacts with the VPS22 component of the ESCRT-II complex. Biochem Biophys Res Commun 2006, 347(4):1074-1079.

doi:10.1186/1471-2121-13-33

Cite this article as: Carrasquillo et al: SNF8, a member of the ESCRT-II complex, interacts with TRPC 6 and enhances its channel activity. BMC Cell Biology 2012 13:33.

\section{Submit your next manuscript to BioMed Central and take full advantage of:}

- Convenient online submission

- Thorough peer review

- No space constraints or color figure charges

- Immediate publication on acceptance

- Inclusion in PubMed, CAS, Scopus and Google Scholar

- Research which is freely available for redistribution 